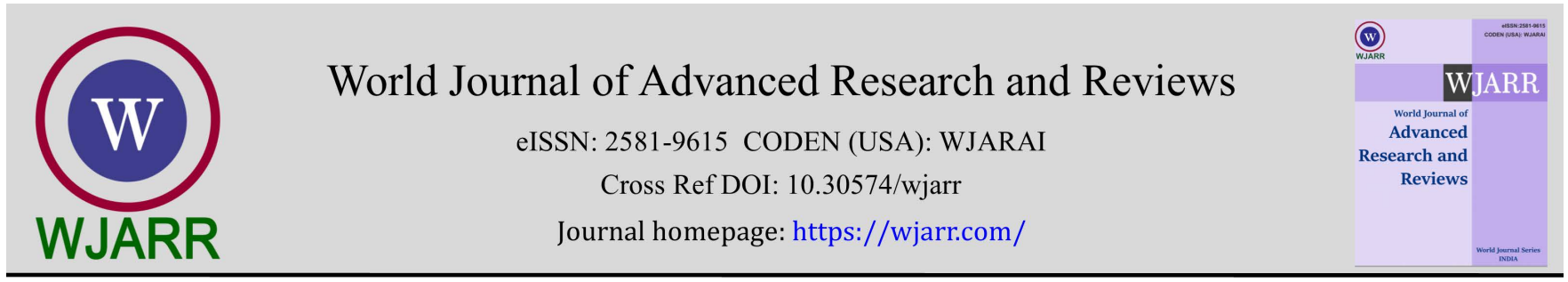

(CASE REPORT)

Check for updates

\title{
Intensity modulated conformal radiotherapy of a jugulo-tympanic paraganglioma
}

\author{
Bouziane Amina *, Mahomed Iyass Yessoufou, Kaoutar Soussy, Hassani Wissal, Farhan Fatima Zahra, Alami \\ Zenab and Bouhafa Touria
}

Department of radiation oncology, University hospital Hassan II, Fez, Morocco.

World Journal of Advanced Research and Reviews, 2021, 12(03), 349-353

Publication history: Received on 15 November 2021; revised on 22 December 2021; accepted on 24 December 2021

Article DOI: https://doi.org/10.30574/wjarr.2021.12.3.0711

\begin{abstract}
Jugulo-tympanic paragangliomas are rare benign tumors arising from the neuroectodermal cells of the tympanic cavity and the dome of the jugular vein. Surgical resection has long been the first-line treatment. However, when this is not possible, radiotherapy remains a therapeutic alternative allowing local control with less morbidity. We report the case of a patient with an unresectable tympano-jugular paraganglioma, the symptomalagy was dominted by chronic headaches and right sided hypoacousie, and the treatment consisted of external radiation therapy with a total dose of 50 Gy in 25 fractions, one fraction per day, 2 Gy per fraction using the IMRT technique. The evolution was good with disappearance of clinical symptomatology, significant local control with less toxicity.
\end{abstract}

Keywords: Jugulo-tympanic; Paraganglioma; IMRT; Radiotheray

\section{Introduction}

Jugulo-tympanic paragangliomas are rare benign tumors arising from the neuroectodermal cells of the tympanic cavity and the dome of the jugular vein [1,2]. Surgical resection has long been the first-line treatment [3]. However, when this is not possible, radiotherapy remains a therapeutic alternative allowing local control with less morbidity [4]. We report the case of a patient with an unresectable tympano-jugular paraganglioma treated with external conformal radiotherapy with intensity modulation.

\section{Observation}

Mrs BM, a 49-year-old patient with no previous pathological history, presented with chronic headaches that were resistant to analgesic treatment, associated with vertigo. The symptomatology worsened with the onset of tinnitus and unilateral right-sided hypoacusis, all evolving in a context of apyrexia and conservation of the general condition.

Otoscopic examination revealed a reddish mass in the right retro-tympanic area and the tone audiometry showed a 40 $\mathrm{dB}$ right conductive hearing loss. lesional process involving the right cerebellopontine angle, centered on the tympanic cavity, with irregular contours, described in T1 hypersignal, T2 heterogeneous hypersignal and flair, containing areas of empty signal in T2, with a typical salt and pepper aspect, and presenting an intense enhancement similar to that of the venous sinuses, testifying to its venous vascular nature, this process measures $24^{*} 28^{*} 60 \mathrm{~mm}$ in diameter, and presents the following relationships and extensions: medially it presents an endocranial extension, coming into contact with the right cerebellar hemisphere and the pons which is slightly deviated to the left, laterally it invades the carotid artery at the level of the siphon, which remains permeable, and infiltrates the paraphryngeal fat, it is responsible for a lysis of the gulf of the jugular vein and

\footnotetext{
* Corresponding author: Bouziane Amina

Department of radiation oncology, University hospital Hassan II, Fez, Morocco.

Copyright $(2021$ Author(s) retain the copyright of this article. This article is published under the terms of the Creative Commons Attribution Liscense 4.0.
} 
the right lateral part of the homolateral occipital bone, below it extends to the upper $1 / 3$ of the cervical portion of the internal jugal vein.

The MRI appearance was in favor of a locally advanced right tympano-jugular paraganglioma with endocranial and parapharyngeal extension.

The patient received external radiation therapy with a total dose of 50 Gy in 25 fractions, one fraction per day, 2 Gy per fraction using the IMRT technique (Figures 2 and 3). The patient received the treatment according to plan and tolerance was good. He was reviewed weekly during treatment by our team. No significant side effects were observed, except for moderate skin reactions on the irradiated skin.

The patient was reviewed after 1 month. The skin reactions had almost disappeared and a disappearance of the symptomatology was noted.
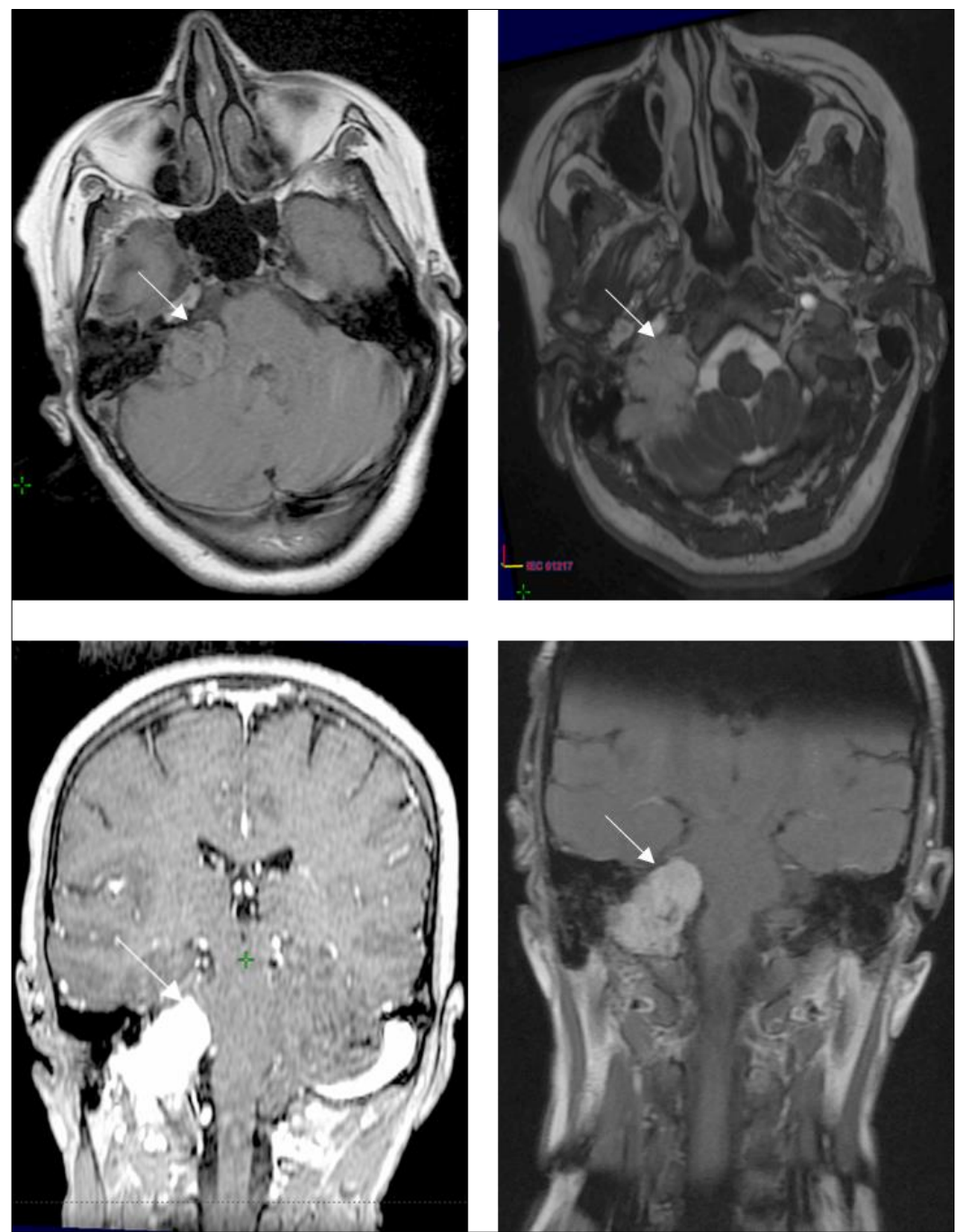

Figure 1 Magnetic resonance imaging (MRI) of the rochers and brain revealed in axial and coronal sections a lesional process involving the right cerebellopontine angle, centered on the tympanic cavity 


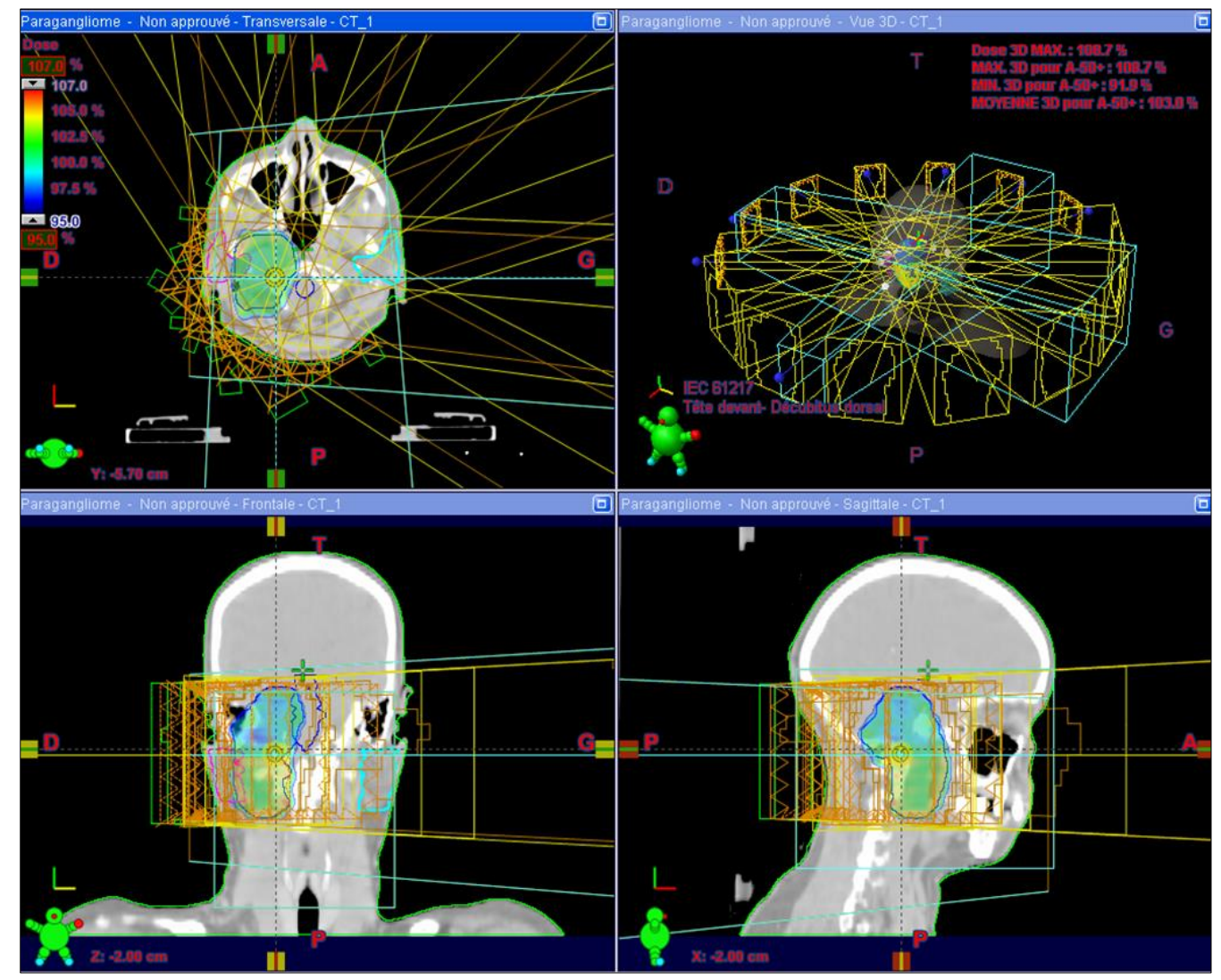

Figure 2 Disposition of multiple IMRT beams on the three plans (axial, sagittal and coronal)

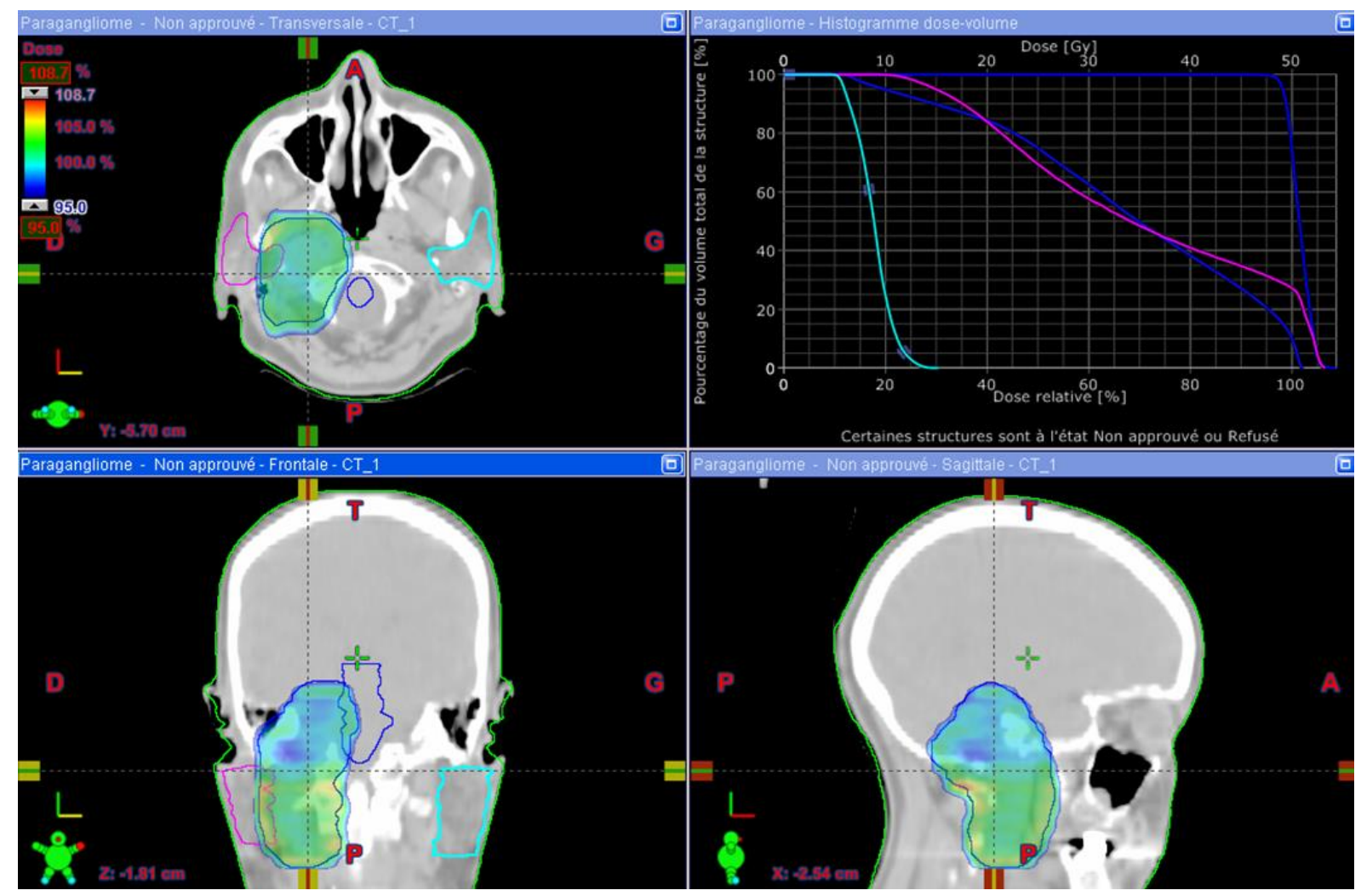

Figure 3 axial, sagittal and coronal view of the prescribed dose to the target volume in blue and dose volume histogram curves 


\section{Discussion}

Paragangliomas are part of a rare entity of neuroendocrine tumors with a low incidence between $1 / 30000$ and $1 / 100000[5,6]$. They represent $0.03 \%$ of all neoplasia and $0.6 \%$ of head and neck tumors $[1,2]$. Most paragangliomas of the cervicofacial region are benign and slow growing. Nevertheless, they can be locally aggressive, invading bone and adjacent soft tissue. Malignant behavior has been demonstrated in less than $5 \%$ of jugulo-tympanic and carotid paragangliomas, in less than $20 \%$ of vagal paragangliomas [7]. Jugular and tympanic paragangliomas present clinically in a similar manner. The onset of symptoms is later in jugular paraganglioma. The most frequent presenting symptom is pulsatile tinnitus, which is present in $80 \%$ of cases. In $60 \%$ of the cases, there is a hearing deficit, of transmission or of perception according to the extension of the tumor. Lesions of the cranial nerves, mainly of pairs IX to XII, or of the sympathetic trunk may result in dysphagia, shoulder weakness or Horner's syndrome [8].

Treatment options for patients with head and neck paragangliomas include surgery, external cortical radiation therapy (RT), stereotactic radiation therapy (SRS), and observation. The choice of treatment depends on the size and location of the tumor, the expected morbidity of treatment, the age of the patient, and patient and physician preferences. Surgery and RT offer a high probability of local control; the main difference between these modalities is the associated level of morbidity, which in turn is related to the location and extent of the tumor. The probability of local control after RT is not related to tumor size or site. A recent review of the literature confirms the efficacy of conformal radiotherapy with or without intensity modulation and stereotactic radiotherapy, both of which achieve tumor control rates ranging from $90 \%$ to almost $100 \%$ of cases, but for different tumor volumes, almost constant stabilization or even improvement of symptoms, and a significantly lower rate of adverse events than with surgery [9].

\section{Conclusion}

Para-lymph node tumors are benign tumors with local evolution. Surgery has been the treatment of choice for a long time, but the sequelae on the cranial nerves and the risk of hemorrhage make it controversial today. Intensity modulated conformal radiotherapy can allow a progressive control of the tumor in 90 to $99 \%$ of cases with a low incidence of minor or reversible complications. It has become the first-line choice.

\section{Compliance with ethical standards}

\section{Acknowledgments}

This research did not receive any specific grant from funding agencies in the public, commercial, or not-for-profit sectors.

\section{Disclosure of conflict of interest}

The authors have no conflicts of interest to disclose.

\section{Statement of ethical approval}

The present research work does not contain any studies performed on animals/humans subjects by any of the authors.

\section{Statement of informed consent}

Informed consent was obtained from all individual participants included in the study.

\section{References}

[1] TYMPANOJUGULAR PARAGANGLIOMAS. TWO CASE REPORTS, vol. 55, no. 2, pp. $339-344$ June 2020 https://doi.org/10.31688/ABMU.2020.55.2.20

[2] R Pialoux, Les paragangliomes de la tête et du cou :À propos de 36 cas, thèse d'exercice, Université de Lorraine, Nancy, France. 1996; 10.4303/ajpm/235986

[3] Jackson CG, McGrew BM, Forest JA, Netterville James L, Hampf Carl F, Glasscock Michael E. Lateral skull base surgery for glomus tumors: long-term control. Otol Neurotol. May 2001; 22(3): 377-82. 
[4] Krych AJ, Foote RL, Brown PD, Yolanda Garces I, Michael Link J. Long-term results of irradiation for paraganglioma. Int J Radiat Oncol Biol Phys. 15 Jul 2006; 65(4): 1063-6.

[5] Capatina C, Ntali G, Karavitaki N, Ashley Grossman B. The management of head-and- neck paragangliomas. Endocr Relat Cancer. 23 Aug 2013; 20(5): R 291-305.

[6] Baysal BE. Hereditary paraganglioma targets diverse paraganglia. J Med Genet. 2002; 39(9): 617-22.

[7] Manolidis S, Shohet JA, Jackson CG, Michael Glasscock E. Malignant glomus tumors. Laryngoscope. Jan 1999; 109(1): 30-4.

[8] Remley KB, Coit WE, Harnsberger HR, Smoker WR, Jacobs JM, McIff EB. Pulsatile tinnitus and the vascular tympanic membrane: CT, MR, and angiographic findings. Radiology. Feb 1990; 174(2): 383-9.

[9] P tran Ba Huy et al Radiotherapy of jugular glomus paraganglioma Eur Ann Otorhinolaryngol Head Neck Dis, September. 2014; 131 (4): 223-6. 